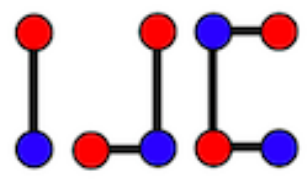

\title{
3-difference cordial labeling of some path related graphs
}

\author{
R. Ponraj ${ }^{\mathrm{a}}$, M. Maria Adaickalam ${ }^{\mathrm{b}}$, R. Kala ${ }^{\mathrm{c}}$ \\ ${ }^{a}$ Department of Mathematics, Sri Paramakalyani College, Alwarkurichi-627412, India. \\ ${ }^{b}$ Research Scholar, Manonmaniam Sundaranar University, Tirunelveli-627012, India. \\ ${ }^{c}$ Manonmaniam Sundaranar University, Tirunelveli-627012, India. \\ ponrajmaths@gmail.com,mariaadaickalam@gmail.com,karthipyi91@yahoo.co.in.
}

\begin{abstract}
Let $G$ be a $(p, q)$-graph. Let $f: V(G) \rightarrow\{1,2, \ldots, k\}$ be a map where $k$ is an integer, $2 \leq k \leq p$. For each edge $u v$, assign the label $|f(u)-f(v)| . f$ is called $k$-difference cordial labeling of $G$ if $\left|v_{f}(i)-v_{f}(j)\right| \leq 1$ and $\left|e_{f}(0)-e_{f}(1)\right| \leq 1$ where $v_{f}(x)$ denotes the number of vertices labelled with $x, e_{f}(1)$ and $e_{f}(0)$ respectively denote the number of edges labelled with 1 and not labelled with 1 . A graph with a $k$-difference cordial labeling is called a $k$-difference cordial graph. In this paper we investigate 3-difference cordial labeling behavior of triangular snake, alternate triangular snake, alternate quadrilateral snake, irregular triangular snake, irregular quadrilateral snake, double triangular snake, double quadrilateral snake, double alternate triangular snake, and double alternate quadrilateral snake.
\end{abstract}

Keywords: Cycle, path, triangular snake, quadrilateral snake, difference cordial.

Mathematics Subject Classification: 05C78

DOI: 10.19184/ijc.2018.2.1.1

\section{Introduction}

Graphs considered in this paper are finite and simple. Graph labeling is used in several areas of science and technology such as coding theory, astronomy, circuit design etc. For more details on application of graph labeling, see [2]. Let $G$ be a $(p, q)$-graph. Let $f: V(G) \rightarrow\{1,2, \ldots, k\}$ be a map. For each edge $u v$, assign the label $|f(u)-f(v)|$. $f$ is called a $k$-difference cordial labeling of $G$ if $\left|v_{f}(i)-v_{f}(j)\right| \leq 1$ and $\left|e_{f}(0)-e_{f}(1)\right| \leq 1$ where $v_{f}(x)$ denotes the number of vertices

Received: 30 Aug 2017, Revised: 23 Dec 2017, Accepted: 07 Feb 2018. 
labelled with $x, e_{f}(1)$ and $e_{f}(0)$ respectively denote the number of edges labelled with 1 and not labelled with 1.

A graph with a $k$-difference cordial labeling is called a $k$-difference cordial graph. The variations of cordial labeling concept was introduced in [1]. R. Hasni et al.[4] investigate the product cordial and total product cordial labeling behavior of some graphs. The notion of difference cordial labeling was introduced by R. Ponraj, S. Sathish Narayanan and R. Kala in [5]. Seoud and Salman [11], studied the difference cordial labeling of some families of graphs, namely, ladder, triangular ladder, grid, step ladder and two sided step ladder graphs etc. Recently, Ponraj et al. [6] introduced the concept of $k$-difference cordial labeling of graphs and studied the 3-difference cordial labeling of star, $m$ copies of star etc. In [7, 8, 9, 10], they discussed the 3-difference cordial labeling of path, cycle, complete graph, complete bipartite graph, star, bistar, comb, double comb, quadrilateral snake, wheel, helms, flower graph, sunflower graph, lotus inside a circle, closed helm, double wheel, union of graphs with the star, union of graphs with splitting graph of star, union of graphs with subdivided star, union of graphs with bistar, $P_{n} \cup P_{n},\left(C_{n} \odot K_{1}\right) \cup\left(C_{n} \odot K_{1}\right), F_{n} \cup F_{n}$, $K_{1, n} \odot K_{2}, P_{n} \odot 3 K_{1}, m C_{4}, \operatorname{spl}\left(K_{1, n}\right), D S\left(B_{n, n}\right), C_{n} \odot K_{2}, C_{4}^{(t)}, S\left(K_{1, n}\right), S\left(B_{n, n}\right), D A\left(T_{n}\right) \odot K_{1}$, $D A\left(T_{n}\right) \odot 2 K_{1}, D A\left(T_{n}\right) \odot K_{2}, D A\left(Q_{n}\right) \odot K_{1}, D A\left(Q_{n}\right) \odot 2 K_{1}$ and some more graphs. In this paper we investigate 3 -difference cordial labeling of triangular snake, alternate triangular snake, alternate quadrilateral snake, irregular triangular snake, irregular quadrilateral snake, double triangular snake, double quadrilateral snake, double alternate triangular snake, and double alternate quadrilateral snake. Terms are not defined here follows from Harary [3].

\section{3-Difference cordial labeling}

We investigate the 3-difference cordial labeling of some path related graphs. The triangular snake $T_{n}$ is obtained from the path $P_{n}$ by replacing each edge of the path by a triangle $C_{3}$.

Theorem 2.1. If $n \equiv 0,1,2(\bmod 4)$ then the triangular snake $T_{n}$ is a 3-difference cordial graph.

Proof. Let $P_{n}$ be a path $u_{1} u_{2} \ldots u_{n}$. Let $V\left(T_{n}\right)=V\left(P_{n}\right) \cup\left\{v_{i}: 1 \leq i \leq n-1\right\}$ and $E\left(T_{n}\right)=$ $E\left(P_{n}\right) \cup\left\{u_{i} v_{i}, v_{i} u_{i+1}: 1 \leq i \leq n-1\right\}$. In this graph $T_{n},\left|V\left(T_{n}\right)\right|=2 n-1$ and $\left|E\left(T_{n}\right)\right|=3 n-3$. Case 1. $n \equiv 0(\bmod 4)$.

Considering the vertices $v_{i}$, assign the label 2 to the vertices $v_{1}, v_{5}, v_{9}, \ldots$. Next assign the label 3 to the vertices $v_{2}, v_{6}, v_{10}, \ldots$. Then we assign the label 1 to the vertices $v_{3}, v_{7}, v_{11}, \ldots$ Now we consider the vertices $v_{4 i}$. Assign the label 1 to the vertices $v_{4 i}$ for the values $i=2,5,8, \ldots$ Next we assign the label 2 to the vertices $v_{4 i}$ for $i=3,6,9, \ldots$ Then for the values $i=1,4,7, \ldots$ assign the label 3 to the vertices $u_{4 i}$. Next we move to the vertices $u_{i}$. Now We consider the vertices $u_{i}$. Assign the label 1 on the vertices $u_{2}, u_{6}, u_{10}, \ldots$ Next assign the label 3 to the vertices $u_{3}, u_{7}, u_{11}, \ldots$ Then assign the label 2 to the vertices $u_{4}, u_{8}, u_{12}, \ldots$ Next we consider the vertices $u_{4 i+1}$, assign the label 1 to the vertices $u_{4 i+1}$ for the values $i=0,3,6, \ldots$ Then assign the label 2 to the vertices $u_{4 i+1}$ for $i=1,4,7, \ldots$ Then for the values $i=2,5,8, \ldots$ assign the label 3 to the vertices $u_{4 i+1}$.

Case 2. $n \equiv 1(\bmod 4)$.

Consider the vertices $v_{i}$. Assign the label 2 to the vertices $v_{2}, v_{6}, v_{10}, \ldots$ Next assign the label 3 to the vertices $v_{3}, v_{7}, v_{11}, \ldots$ Then assign the label 1 to the vertices $v_{4}, v_{8}, v_{12}, \ldots$ Now we consider the vertices $v_{4 i+1}$. Assign the label 1 to the vertices $v_{4 i+1}$ for the values $i=0,3,6, \ldots$ Then assign the 
label 2 to the vertices $v_{4 i+1}$ for $i=1,4,7, \ldots$ Then for the values $i=2,5,8, \ldots$ assign the label 3 to the vertices $v_{4 i+1}$. Next we move to the path vertices $u_{i}$. First we fix the label 2 to the vertex $u_{1}$. Now we consider the vertices $u_{i}$ as in the label 1 to the vertices $u_{3}, u_{7}, u_{11}, \ldots$ Next we assign the label 3 to the vertices $u_{4}, u_{8}, u_{12}, \ldots$ Then we assign the label 2 to the vertices $u_{5}, u_{9}, u_{13}, \ldots$ Now we consider the vertices $u_{4 i+2}$. Assign the label 3 to the vertices $u_{4 i+2}$ for the values $i=0,3,6, \ldots$ Then assign the label 1 to the vertices $u_{4 i+2}$ for $i=1,4,7, \ldots$ Then for the values $i=2,5,8, \ldots$ assign the label 2 to the vertices $u_{4 i+2}$.

Case 3. $n \equiv 2(\bmod 4)$.

First we fix the label 1 to the vertex $v_{1}$. Now we consider the vertices $v_{i}$ as in the label 2 to the vertices $v_{3}, v_{7}, v_{11}, \ldots$ Next assign the label 3 to the vertices $v_{4}, v_{8}, v_{12}, \ldots$ then assign the label 1 to the vertices $v_{5}, v_{9}, v_{13}, \ldots$ We now consider the vertices $v_{4 i+2}$. Assign the label 1 to the vertices $v_{4 i+2}$ for the values $i=0,3,6, \ldots$ Next we assign the label 2 to the vertices $v_{4 i+2}$ for $i=1,4$, $7, \ldots$ Then for the values $i=2,5,8, \ldots$ assign the label 3 to the vertices $v_{4 i+2}$. Next we move to the path vertices $u_{i}$. Now we fix the labels 2 and 3 to the vertices $u_{1}$ and $u_{2}$ respectively. Next we consider the vertices $u_{i}$. Assign the label 1 to the vertices $u_{4}, u_{8}, u_{12}, \ldots$ Next assign the label 3 to the vertices $u_{5}, u_{9}, u_{13}, \ldots$ Then assign the label 2 to the vertices $u_{6}, u_{10}, u_{14}, \ldots$ Next we consider the vertices $u_{4 i+3}$. Assign the label 2 to the vertices $u_{4 i+3}$ for the values $i=0,3,6, \ldots$ Next assign the label 3 to the vertices $u_{4 i+3}$ for $i=1,4,7, \ldots$ Then for the values $i=2,5,8, \ldots$ assign the label 1 to the vertices $u_{4 i+3}$. The vertex and edge condition are given in table 1 and 2 respectively.

\begin{tabular}{|l|l|l|l|}
\hline Value of $\mathrm{n}$ & $v_{f}(1)$ & $v_{f}(2)$ & $v_{f}(3)$ \\
\hline$n \equiv 2,5,8(\bmod 12)$ & $\frac{2 n-1}{3}$ & $\frac{2 n-1}{3}$ & $\frac{2 n-1}{3}$ \\
\hline$n \equiv 4(\bmod 12)$ & $\frac{2 n+1}{3}$ & $\frac{2 n-2}{3}$ & $\frac{2 n-2}{3}$ \\
\hline$n \equiv 0(\bmod 12)$ & $\frac{2 n}{3}$ & $\frac{2 n-3}{3}$ & $\frac{2 n}{3}$ \\
\hline$n \equiv 6,9(\bmod 12)$ & $\frac{2 n}{3}$ & $\frac{2 n}{3}$ & $\frac{2 n-3}{3}$ \\
\hline$n \equiv 1,10(\bmod 12)$ & $\frac{2 n-2}{3}$ & $\frac{2 n+1}{3}$ & $\frac{2 n-2}{3}$ \\
\hline
\end{tabular}

Table 1. Vertex label

\begin{tabular}{|l|l|l|}
\hline values of $n$ & $e_{f}(0)$ & $e_{f}(1)$ \\
\hline$n \equiv 0(\bmod 4)$ & $\frac{3 n-2}{2}$ & $\frac{3 n-4}{2}$ \\
\hline$n \equiv 1(\bmod 4)$ & $\frac{3 n-3}{2}$ & $\frac{3 n-3}{2}$ \\
\hline$n \equiv 2(\bmod 4)$ & $\frac{3 n-4}{2}$ & $\frac{3 n-2}{2}$ \\
\hline
\end{tabular}

Table 2. Edge labels 


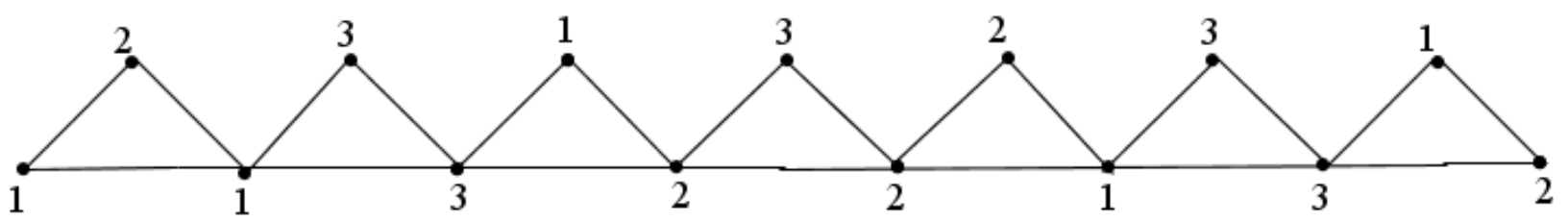

Figure 1. A 3-difference cordial labeling for $T_{8}$

Next is the alternate triangular snake. An alternate triangular snake $A T_{n}$ is obtained from a path $u_{1} u_{2} \ldots u_{n}$ by joining $u_{i}$ and $u_{i+1}$ (alternatively) to new vertex $v_{i}$. That is every alternate edge of a path is replaced by $C_{3}$.

Theorem 2.2. Alternate triangular snakes are 3-difference cordial graphs.

Proof. Case 1. Let the first triangle starts from $u_{2}$ and the last triangle be ends with $u_{n-1}$. In this case $\left|V\left(A T_{n}\right)\right|=\frac{3 n-2}{2}$ and $\left|E\left(A T_{n}\right)\right|=2 n-3$. Assign label 2 to the vertices $v_{1}, v_{2}, \ldots$ We assign the label to the path vertices $u_{1}, u_{2}, \ldots u_{n}$ in the pattern $1,3,1,3, \ldots .1,3$. Note that in this process the vertex $u_{n}$ and $u_{n-1}$ received the labels 1,3 respectively. In this case the vertex condition is given by $v_{f}(1)=v_{f}(3)=\frac{n}{2}$ and $v_{f}(2)=\frac{n}{2}-1$. Also the edge condition is $e_{f}(0)=n-1$ and $e_{f}(1)=n-2$.

Case 2. Let the first triangle starts from $u_{1}$ and the last triangle be ends with $u_{n}$. Here $\left|V\left(A T_{n}\right)\right|=\frac{3 n}{2}$ and $\left|E\left(A T_{n}\right)\right|=2 n-1$. Assign the label to the vertices as in case 1 . The vertex and edge conditions are given by $v_{f}(1)=v_{f}(2)=v_{f}(3)=\frac{n}{2}$ and $e_{f}(0)=n-1$ and $e_{f}(1)=n$ respectively.

Case 3. Let the first triangle starts from $u_{2}$ and the last triangle be ends with $u_{n}$. Note that in this case $\left|V\left(A T_{n}\right)\right|=\frac{3 n-1}{2}$ and $\left|E\left(A T_{n}\right)\right|=2 n-2$. Assign the label to the vertices as in case 1 . It is easy to verify that the last vertex $u_{n}$ received the label 1 in this case. This vertex labeling is a 3 -difference cordial labeling follows from the vertex and edge condition $v_{f}(1)=\frac{n+1}{2}$, $v_{f}(2)=v_{f}(3)=\frac{n-1}{2}$ and $e_{f}(0)=e_{f}(1)=n-1$.

Case 4. Let the first triangle starts from $u_{1}$ and the last triangle be ends with $u_{n-1}$.

This case is equivalent to case 3 .

Now we look into alternate quadrilateral snake. An alternate quadrilateral snake $A Q_{n}$ is obtained from a path $u_{1} u_{2} \ldots u_{n}$ by joining $u_{i}, u_{i+1}$ (alternatively) to new vertices $v_{i}, w_{i}$ respectively and then joining $v_{i}$ and $w i$. That is every alternate edge of a path is replaced by a cycle $C_{4}$.

Theorem 2.3. All alternate quadrilateral snakes are 3-difference cordial graphs.

Proof. Case 1. Let the first $C_{4}$ be starts from $u_{2}$ and the last $C_{4}$ be ends with $u_{n-1}$.

Note that in this case $\left|V\left(A Q_{n}\right)\right|=2 n-2$ and $\left|E\left(A Q_{n}\right)\right|=\frac{5 n-8}{2}$. We consider the vertices in the path. Assign the labels $2,1,1,3$ to the vertices $u_{1}, u_{2}, u_{3}, u_{4}$. Next we assign the labels 2,1 , 1,3 to the next four vertices $u_{5}, u_{6}, u_{7}, u_{8}$. Continuing this way, assign the label to the next four vertices and so on. Clearly in this process the vertex $u_{n}$ received the labels 1 or 3 according as $n \equiv 2(\bmod 4)$ or $n \equiv 0(\bmod 4)$. Then we move to the vertices $v_{i}$ and $w_{i}$. Fix the labels 2 and 3 to the vertices $v_{1}$ and $w_{1}$ respectively. Assign the labels 1 and 3 to the vertices $v_{12 i+2}$ and $w_{12 i+2}$ 
for the values $i=0,1,2,3, \ldots$ Then assign the label 2 to the vertices $v_{12 i+3}$ and $w_{12 i+3}$ for $i=0,1$, $2,3, \ldots$ For the values of $i=0,1,2,3, \ldots$ assign the label 3 to the vertices $v_{12 i+4}$ and $w_{12 i+4}$. Next assign the labels 2 and 3 to the vertices $v_{6 i+5}$ and $w_{6 i+5}$ for all the vertices of $i=0,1,2,3 \ldots$ Then we assign the labels 1 and 3 to the vertices $v_{6 i}$ and $w_{6 i}$ for $i=1,2,3, \ldots$ For all the values of $i=1$, $2,3, \ldots$ assign the label 2 to the vertices $v_{6 i+1}$ and $w_{6 i+1}$ respectively. Now we assign the label 3 to the vertices $v_{12 i+8}$ and $w_{12 i+8}$ for all the values of $i=0,1,2,3, \ldots$ Then assign the labels 2 and 1 to the vertices $v_{12 i+9}$ and $w_{12 i+9}$ for $i=0,1,2,3, \ldots$ For the values $i=0,1,2,3, \ldots$ assign the labels 3 and 2 to the vertices $v_{12 i+10}$ and $w_{12 i+10}$ respectively. Clearly Clearly the vertex and edge label satisfy the condition of this case are given in table 3 and table 4.

\begin{tabular}{|l|l|l|l|}
\hline Value of $\mathrm{n}$ & $v_{f}(1)$ & $v_{f}(2)$ & $v_{f}(3)$ \\
\hline$n \equiv 4,10,16,22(\bmod 24)$ & $\frac{2 n-2}{3}$ & $\frac{2 n-2}{3}$ & $\frac{2 n-2}{3}$ \\
\hline$n \equiv 6(\bmod 24)$ & $\frac{2 n}{3}$ & $\frac{2 n-3}{3}$ & $\frac{2 n-3}{3}$ \\
\hline$n \equiv 8(\bmod 24)$ & $\frac{2 n-1}{3}$ & $\frac{2 n-1}{3}$ & $\frac{2 n-4}{3}$ \\
\hline$n \equiv 0,12,18(\bmod 24)$ & $\frac{2 n-3}{3}$ & $\frac{2 n-3}{3}$ & $\frac{2 n}{3}$ \\
\hline
\end{tabular}

Table 3. Vertex label

\begin{tabular}{|l|l|l|}
\hline values of $\mathrm{n}$ & $e_{f}(0)$ & $e_{f}(1)$ \\
\hline$n \equiv 0(\bmod 4)$ & $\frac{5 n-8}{4}$ & $\frac{5 n-8}{4}$ \\
\hline$n \equiv 2(\bmod 4)$ & $\frac{5 n-10}{4}$ & $\frac{5 n-6}{4}$ \\
\hline
\end{tabular}

Table 4. Edge label

Case 2. Let the first $C_{4}$ be starts from $u_{1}$ and the last $C_{4}$ be ends with $u_{n}$. Here $\left|V\left(A\left(Q_{n}\right)\right)\right|=2 n$ and $\left|E\left(A\left(Q_{n}\right)\right)\right|=\frac{5 n-2}{2}$. First we consider the vertices of a path. Assign the labels $1,3,2,1$ to the first four vertices $u_{1}, u_{2}, u_{3}, u_{4}$ respectively. Next we assign the labels 1 , $3,2,1$ to the next four vertices $u_{5}, u_{6}, u_{7}, u_{8}$ respectively. Proceeding like this, assign the next four vertices and so on. Clearly the vertex $u_{n}$ received the label 1 or 3 according as $n \equiv 0(\bmod 4)$ or $n \equiv 2(\bmod 4)$. Consider the vertices $v_{6 i+2}$ and $v_{6 i+4}$. Now we assign the label 3 to the vertices $v_{6 i+2}$ and $v_{6 i+4}$ for all the values of $i=0,1,2,3, \ldots$ Assign the label 3 to the vertices $w_{2 i}$ for $i=1$, $2,3, \ldots$ Next we assign the label 2 to the vertice $v_{6 i+3}$ and $v_{6 i+}$ for all the values of $i=0,1,2,3, \ldots$ For all the vvalues of $i=1,2,3, \ldots$ assign the label 2 to the vertices $w_{2 i+1}$. Now we consider the vertices $v_{6 i}$ and $v_{6 i+1}$ for all the vaalues $i=1,2,3, \ldots$ The vertex and edge condition of this case are given in table 5 and tablet6.

\begin{tabular}{|l|l|l|l|}
\hline Value of $\mathrm{n}$ & $v_{f}(1)$ & $v_{f}(2)$ & $v_{f}(3)$ \\
\hline$n \equiv 0,6(\bmod 12)$ & $\frac{2 n}{3}$ & $\frac{2 n}{3}$ & $\frac{2 n}{3}$ \\
\hline$n \equiv 4(\bmod 12)$ & $\frac{2 n+1}{3}$ & $\frac{2 n-2}{3}$ & $\frac{2 n+1}{3}$ \\
\hline$n \equiv 10(\bmod 12)$ & $\frac{2 n-2}{3}$ & $\frac{2 n+1}{3}$ & $\frac{2 n+1}{3}$ \\
\hline$n \equiv 2(\bmod 12)$ & $\frac{2 n+2}{3}$ & $\frac{2 n-1}{3}$ & $\frac{2 n-1}{3}$ \\
\hline
\end{tabular}

Table 5. Vertex label 


\begin{tabular}{|l|l|l|}
\hline values of $\mathrm{n}$ & $e_{f}(0)$ & $e_{f}(1)$ \\
\hline$n \equiv 0(\bmod 4)$ & $\frac{5 n-4}{4}$ & $\frac{5 n}{4}$ \\
\hline$n \equiv 2(\bmod 4)$ & $\frac{5 n-2}{4}$ & $\frac{5 n-2}{4}$ \\
\hline
\end{tabular}

Table 6. Edge label

Case 3. Let the first $C_{4}$ be starts from $u_{2}$ and the last $C_{4}$ be ends with $u_{n}$.

Note that $\left|V\left(A\left(Q_{n}\right)\right)\right|=2 n-1$ and $\left|E\left(A\left(Q_{n}\right)\right)\right|=\frac{5 n-5}{2}$. First we fix the label 1 to the vertex $u_{1}$. Then we consider the path vertices $u_{i}$ as in the labels $1,3,2,1$ to the four vvertices $u_{2}, u_{3}, u_{4}, u_{5}$ respectively. Next assign the labels $1,3,2,1$ to the net four vertices $u_{6}, u_{7}, u_{8}, u_{9}$ respectively. Continuing like this to assign the label to the next four vertices and so on. In this process, the last vertex $u_{n}$ received the label 3 or 1 according as $n \equiv 3(\bmod 4)$ or $n \equiv 1(\bmod 4)$. Consider the vertices $v_{6 i+1}$ and $v_{6 i+3}$. Assign the label 2 to the vertices $v_{6 i+1}$ and $v_{6 i+3}$ for the values $i=0,1,2$, $3, \ldots$ Next assign the label 3 to the vertices $v_{6 i+2}$ and $v_{6 i+4}$ for $i=0,1,2, \ldots$ For the values of $i=0$, $1,2, \ldots$. assign the label 1 to the vertices $v_{6 i+5}$. Then assign the label 1 to the vertices $v_{6 i}$ for $i=1$, $2,3, \ldots$ Now we move to the vertices $w_{i}$. Assign the label 2 to the vertices $w_{2 i+1}$ for all the values of $i=0,1,2,3, \ldots$ Then assign the label 3 to the vertices $w_{2 i}$ for $i=1,2,3, \ldots$ Clearly the vertex and edge condition of this case are given in table 7 and table 8.

\begin{tabular}{|l|l|l|l|}
\hline Value of $\mathrm{n}$ & $v_{f}(1)$ & $v_{f}(2)$ & $v_{f}(3)$ \\
\hline$n \equiv 5,11(\bmod 12)$ & $\frac{2 n-1}{3}$ & $\frac{2 n-1}{3}$ & $\frac{2 n-1}{3}$ \\
\hline$n \equiv 3(\bmod 12)$ & $\frac{2 n}{3}$ & $\frac{2 n}{3}$ & $\frac{2 n-3}{3}$ \\
\hline$n \equiv 7(\bmod 12)$ & $\frac{2 n-2}{3}$ & $\frac{2 n+1}{3}$ & $\frac{2 n-2}{3}$ \\
\hline$n \equiv 9(\bmod 12)$ & $\frac{2 n-3}{3}$ & $\frac{2 n}{3}$ & $\frac{2 n}{3}$ \\
\hline$n \equiv 1(\bmod 12)$ & $\frac{2 n+1}{3}$ & $\frac{2 n-2}{3}$ & $\frac{2 n-2}{3}$ \\
\hline
\end{tabular}

Table 7. Vertex label

\begin{tabular}{|l|l|l|}
\hline values of $\mathrm{n}$ & $e_{f}(0)$ & $e_{f}(1)$ \\
\hline$n \equiv 1(\bmod 4)$ & $\frac{5 n-5}{4}$ & $\frac{5 n-5}{4}$ \\
\hline$n \equiv 3(\bmod 4)$ & $\frac{5 n-3}{4}$ & $\frac{5 n-7}{4}$ \\
\hline
\end{tabular}

Table 8. Edge label

Case 4. Let the first $C_{4}$ be starts from $u_{1}$ and the last $C_{4}$ be ends with $u_{n-1}$. This case is equivalent to case 3 .

Next investigation is about the irregular triangular snakes. The irregular triangular snake $I T_{n}$ is obtained from the path $u_{1} u_{2} \ldots u_{n}$ with vertex set $V\left(I T_{n}\right)=V\left(P_{n}\right) \cup\left\{v_{i}: 1 \leq i \leq n-2\right\}$ and the edge set $E\left(I T_{n}\right)=E\left(P_{n}\right) \cup\left\{u_{i} v_{i}, v_{i} u_{i+2}: 1 \leq i \leq n-2\right\}$.

Theorem 2.4. The irregular triangular snake is a 3-difference cordial graph.

Proof. Clearly $\left|V\left(I T_{n}\right)\right|=2 n-2$ and $\left|E\left(I T_{n}\right)\right|=3 n-5$. Let $n=3 t+r, 0 \leq r<8$. Assign the labels $1,3,2,2,3,1,2,2$ to the first eight path vertices. Next eight path vertices are labeled 
by $1,3,2,2,3,1,2,2$. Proceeding like this, assign the label to the $8 \mathrm{t}$ vertices of the path. Note that the vertex $u_{8 t}$ receive the label 2 . Assign the label to the next $r$ vertices $u_{8 t+1}, u_{8 t+2}, \ldots, u_{8 t+r}$ by the sequence of integers $1,3,2,2,3,1, \ldots$. Next assign the label to the first five vertices $v_{1}, v_{2}, v_{3}, v_{4}, v_{5}$ by the integers $1,3,2,1,3$. Then assign the labels $1,3,2,1,3$ to the next five vertices $v_{6}, v_{7}, v_{8}, v_{9}, v_{10}$. Continuing this way, assign the label to the next five vertices and so on. If all the vertices $v_{i}$ are labeled, then stop. Otherwise there are some non labeled vertices and the number of labeled verties is less than or equal to 4 . Assign the labels 1, 3, 2, 1 to the non labeled vertices. That is if only one non labeled vertices is exists we use the label 1 only if it is two then we use the labels 1, 3 and so on. This labeling is clearly 3 -difference cordial labeling follows from table 9.

\begin{tabular}{|l|l|l|}
\hline Value of $\mathrm{n}$ & $e_{f}(0)$ & $e_{f}(1)$ \\
\hline$n$ is odd & $\frac{3 n-5}{2}$ & $\frac{3 n-5}{2}$ \\
\hline$n$ is even & $\frac{3 n-4}{2}$ & $\frac{3 n-6}{2}$ \\
\hline
\end{tabular}

Table 9. Edge label

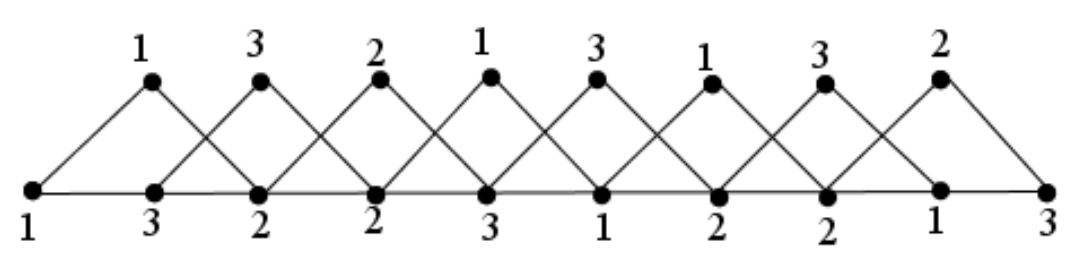

Figure 2. A 3-difference cordial labeling of irregular triangular snake $I T_{10}$

The irregular quadrilateral snake $I Q_{n}$ is obtained from the path $P_{n}: u_{1} u_{2} \ldots u_{n}$ with vertex set $V\left(I Q_{n}\right)=V\left(P_{n}\right) \cup\left\{v_{i}, w_{i}: 1 \leq i \leq n-2\right\}$ and the edge set $E\left(I Q_{n}\right)=E\left(P_{n}\right) \cup\left\{u_{i} v_{i}, w_{i} u_{i+2}\right.$ : $1 \leq i \leq n-2\}$.

Theorem 2.5. The irregular quadrilateral snake is a 3-difference cordial graph.

Proof. Clearly $\left|V\left(I Q_{n}\right)\right|=3 n-4$ and $\left|E\left(I Q_{n}\right)\right|=4 n-7$ respectively. We consider the path vertices $u_{i}$. Assign the labels $1,2,3$ to the first three path vertices $u_{1}, u_{2}, u_{3}$ respectively. Then assign the label 1 to the remaining path vertices $u_{4}, u_{5}, u_{6}, \ldots$ Next assign the label 2 to the vertices $v_{i}$ and assign the label 3 to the vertices $w_{i}$. Clearly $e_{f}(0)=2 n-4$ and $e_{f}(1)=n-2$ and $v_{f}(1)=n-1$ and $v_{f}(2)=v_{f}(3)=n-1$. Therefore $I Q_{n}$ is 3-difference cordial.

A double triangular snake $D T_{n}$ consists of two triangular snakes that have a common path. That is a double triangular snake is obtained from a path $u_{1} u_{2} \ldots u_{n}$ by joining $u_{i}$ and $u_{i+1}$ to a new vertex $v_{i}(1 \leq i \leq n-1)$ and to a new vertex $w_{i}(1 \leq i \leq n-1)$.

Theorem 2.6. Double triangular snake $D T_{n}$ is a 3-difference cordial graph. 
Proof. Here $\left|V\left(D T_{n}\right)\right|=3 n-2$ and $\left|E\left(D T_{n}\right)\right|=5 n-5$. First we consider the path vertices $u_{i}$. Assign the labels 1,3 to the vertices $u_{1}, u_{2}$ respectively. Then assign the labels $2,3,3,3$ to the path vertices $u_{3}, u_{4}, u_{5}, u_{6}$. Using the same pattern assign the labels $2,3,3,3$ to the next four path vertices $u_{7}, u_{8}, u_{9}, u_{10}$. Continuing this way assign the next four vertices and so on. If all the path vertices are labeled in this way then we stop the process. Otherwise there are some non labeled vertices exists and in the case the number of non labeled vertices less than or equal to 3 . Assign the labels 2, 3, 3 to the non labeled vertices. If only one non labeled vertex exists then we use the label 2 only. If there are two labeled vertices then we use the labels 2,3 . Next we move to the vertices $v_{i}, w_{i}$. Assign the label 1 to all the vertices $v_{i}(1 \leq i \leq n-1)$. Assign the label 2 to the vertex $w_{1}$. Then assign the labels $3,2,2,2$ to the four vertices $w_{2}, w_{3}, w_{4}, w_{5}$. Next we assign the labels $3,2,2,2$ to the next four vertices $w_{6}, w_{7}, w_{8}, w_{9}$. Proceeding like this, if all the vertices $w_{i}$ are labeled then stop. Otherwise next non labeled vertices in the sequence $3,2,2$. This labeling is a 3 -difference cordial labeling and its vertex and edge condition is given by $v_{f}(1)=n$ and $v_{f}(2)=v_{f}(3)=n-1$.

\begin{tabular}{|l|l|l|}
\hline Value of $\mathrm{n}$ & $e_{f}(0)$ & $e_{f}(1)$ \\
\hline$n$ is odd & $\frac{5 n-5}{2}$ & $\frac{5 n-5}{2}$ \\
\hline$n$ is even & $\frac{5 n-4}{2}$ & $\frac{5 n-6}{2}$ \\
\hline
\end{tabular}

Table 10. Edge label

A double quadrilateral snake $D Q_{n}$ consists of two quadrilateral snake have a common path.

Theorem 2.7. The double quadrilateral snake is a 3-difference cordial graph.

Proof. Let $V\left(D Q_{n}\right)=\left\{u_{i}: 1 \leq i \leq n\right\} \cup\left\{v_{i}, w_{i}, x_{i}, y_{i}: 1 \leq i \leq n-1\right\}$ and $E\left(D Q_{n}\right)=$ $\left\{u_{i} u_{i+1}, u_{i} v_{i}, v_{i} w_{i}, u_{i} x_{i}, x_{i} y_{i}, w_{i} u_{i+1}, y_{i} u_{i+1}: 1 \leq i \leq n-1\right\}$. Clearly $\left|V\left(D Q_{n}\right)\right|=5 n-4$ and $\left|E\left(D Q_{n}\right)\right|=7 n-7$. Consider the path vertices $u_{i}$. Assign the labels $1,1,2,2$ to the first four vertices $u_{1}, u_{2}, u_{3}, u_{4}$ respectively. Then assign the labels $1,1,2,2$ to the next four vertices $u_{5}, u_{6}, u_{7}, u_{8}$. Proceeding like this, assign the next four vertices and so on. If all the path vertices are labeled in this way then the labelling is complete. Otherwise there are some non labeled vertices exist. If the number of non labeled vertices less than or equal to 3 , then assign the labels $1,1,2$ to the non labeled vertices. If only one non labeled vertex exists then we use the label 1 only. If there are two unlabelled vertices, then we use the labels 1,1 . Now we move to the vertices $v_{i}$ and $w_{i}$. Assign the labels 2, 1, 3, 2 to the vertices $v_{1}, v_{2}, v_{3}, v_{4}$ respectively. Now we assign the labels $3,1,2,1$ to the vertices $w_{1}, w_{2}, w_{3}, w_{4}$ respectively. Next assign the label 2 to the vertices $v_{12 i+5}, v_{12 i+7}, v_{12 i+9}, v_{12 i+11}$ for all the values of $i=0,1,2,3, \ldots$. Then assign the label 1 to the vertices $w_{12 i+5}, w_{12 i+6}, w_{12 i+9}, w_{12 i+10}$ for $i=0,1,2,3, \ldots$. Now for all the values of $i=1,2,3, \ldots$ assign the label 2 to the vertices $v_{12 i+1}, v_{12 i+3}, v_{12 i+4}$. Next we assign the label 1 to the vertices $w_{12 i+1}, w_{12 i+2}, w_{12 i+4}$ for all the values $i=0,1,2,3, \ldots$. Then we assign the label 1 to the vertices $v_{12 i+6}, v_{12 i+10}$ for all the values of $i=0,1,2,3, \ldots$. For all the values of $i=0,1,2,3, \ldots$ assign the label 3 to the vertices $w_{12 i+7}, w_{12 i+8}, w_{12 i+11}$. Now assign the label 1 to the vertices $v_{12 i}, v_{12 i+2}$ for all the values of $i=0,1,2,3, \ldots$. Next assign the label 3 to the vertices $w_{12 i}, w_{12 i+3}$ for all 
the values of $i=0,1,2,3, \ldots$. We consider the vertices $x_{i}$ and $y_{i}$. Assign the labels $2,3,1,2$ to the vertices $x_{1}, x_{2}, x_{3}, x_{4}$ respectively. Then we assign the labels $2,3,1,2$ to the next four vertices $x_{5}, x_{6}, x_{7}, x_{8}$ respectively. Continuing this way we assign the next four vertices and so on. If all the vertices are labeled in this way, then stop. Otherwise there are some non labeled vertices exist. If the number of non labeled vertices less than or equal to 3 , then assign the labels $2,3,1$ to the non labeled vertices. If only one unlabeled vertex exists, then we use the label 2 only. If there are two then we use the labels 2,3 . Now we assign the label 3 to all the vertices $y_{i}(1 \leq i \leq n-1)$. This labeling is 3 -difference cordial labeling follows from the following tables.

\begin{tabular}{|l|l|l|}
\hline values of $\mathrm{n}$ & $e_{f}(0)$ & $e_{f}(1)$ \\
\hline$n \equiv 1(\bmod 2)$ & $\frac{7 n-7}{2}$ & $\frac{7 n-7}{2}$ \\
\hline$n \equiv 0(\bmod 2)$ & $\frac{7 n-8}{2}$ & $\frac{7 n-6}{2}$ \\
\hline
\end{tabular}

Table 11. Edge label

\begin{tabular}{|l|l|l|l|}
\hline Value of $\mathrm{n}$ & $v_{f}(1)$ & $v_{f}(2)$ & $v_{f}(3)$ \\
\hline$n \equiv 2,5,8,11(\bmod 12)$ & $\frac{5 n-4}{3}$ & $\frac{5 n-4}{3}$ & $\frac{5 n-4}{3}$ \\
\hline$n \equiv 3(\bmod 12)$ & $\frac{5 n-3}{3}$ & $\frac{5 n-6}{3}$ & $\frac{5 n-3}{3}$ \\
\hline$n \equiv 7(\bmod 12)$ & $\frac{5 n-2}{3}$ & $\frac{5 n-5}{3}$ & $\frac{5 n-5}{3}$ \\
\hline$n \equiv 6(\bmod 12)$ & $\frac{5 n-3}{3}$ & $\frac{5 n-3}{3}$ & $\frac{5 n-6}{3}$ \\
\hline$n \equiv 0,9(\bmod 12)$ & $\frac{5 n-6}{3}$ & $\frac{5 n-3}{3}$ & $\frac{5 n-3}{3}$ \\
\hline$n \equiv 10(\bmod 12)$ & $\frac{5 n-5}{3}$ & $\frac{5 n-2}{3}$ & $\frac{5 n-5}{3}$ \\
\hline$n \equiv 1,4(\bmod 12)$ & $\frac{5 n-5}{3}$ & $\frac{5 n-5}{3}$ & $\frac{5 n-2}{3}$ \\
\hline
\end{tabular}

Table 12. Vertex label

A double alternate triangular snake $D A T_{n}$ consists of two alternate triangular snakes that have a common path. That is a double alternate triangular snake is obtained from a path $u_{1} u_{2} \ldots . u_{n}$ by joining $u_{i}$ and $u_{i+1}$ (alternatively) to two vertices $v_{i}$ and $w_{i}$.

Theorem 2.8. Double alternate triangular snake $D A T_{n}$ is a 3-difference cordial graph.

Proof. Case 1. The triangle starts from $u_{1}$ and end with $u_{n}$.

In this case $\left|V\left(D A T_{n}\right)\right|=2 n$ and $\left|E\left(D A T_{n}\right)\right|=3 n-1$. Assign the label 1 to the path vertex $u_{1}$. Now we assign the label 2 to the vertices $u_{12 i+2}, u_{12 i+3}, u_{12 i+6}, u_{12 i+7}, u_{12 i+10}, u_{12 i+11}$ for all the values of $i=0,1,2,3, \ldots$ Then we assign the labels 3 to the vertices $u_{12 i+4}, u_{12 i+5}, u_{12 i+8}, u_{12 i+9}$ for all the values of $i=0,1,2,3, \ldots$ For all the values of $i=0,1,2,3, \ldots$ assign the label 1 to the vertices $u_{12 i}$ and $u_{12 i+1}$. Next we move to the vertices $v_{i}$ and $w_{i}$. Assign the labels $2,1,3,1,2,3$ to the first six vertices $v_{1}, v_{2}, v_{3}, v_{4}, v_{5}, v_{6}$ respectively. Then we assign the labels $2,1,3,1,2,3$ to the next six vertices $v_{7}, v_{8}, v_{9}, v_{10}, v_{11}, v_{12}$ respectively. Proceeding like this assign the label to the next six vertices and so on. If all the vertices $v_{i}$ are labeled then stop the process. Otherwise there are some non labeled vertices and number of labeled vertices is less than or equal to 5 . Now assign the 
labels $2,1,3,1,2$ to the non labeled vertices. If four non labeled vertices are exist then assign the labels $2,1,3,1$ to th non labeled vertices. If the number of non labeled vertices is 3 then assign the labels $2,1,3$ to the non labeled vertices. If only one non labeled vertex exist then assign the label 2 only. If it is two then assign the labels 2,1 to the non labeled vertices. Consider the vertices $w_{i}$. Assign the labels $3,1,1$ to the first three vertices $w_{1}, w_{2}, w_{3}$ respectively. Then we assign the labels $3,1,1$ to the next three vertices $w_{4}, w_{5}, w_{6}$ respectively. Continuing this way we assign the label to the next three vertices and so on. If all the vertices $w_{i}$ are labeled, then stop the process. Otherwise there are some non labeled vertices exists. If the number of non labeled vertices are less than or equal to 2 then assign the labels 3,1 to the non labeled vertices. If only one non labeled vertex is exist then assign the label 3 only. The edge condition is given by $e_{f}(0)=\frac{3 n-2}{2}$ and $e_{f}(1)=\frac{3 n}{2}$. Also the vertex condition is given by a table 13 .

\begin{tabular}{|l|l|l|l|}
\hline Value of $\mathrm{n}$ & $v_{f}(1)$ & $v_{f}(2)$ & $v_{f}(3)$ \\
\hline$n \equiv 0,6(\bmod 12)$ & $\frac{2 n}{3}$ & $\frac{2 n}{3}$ & $\frac{2 n}{3}$ \\
\hline$n \equiv 4(\bmod 12)$ & $\frac{2 n+1}{3}$ & $\frac{2 n+1}{3}$ & $\frac{2 n-2}{3}$ \\
\hline$n \equiv 8(\bmod 12)$ & $\frac{2 n-1}{3}$ & $\frac{2 n-1}{3}$ & $\frac{2 n+2}{3}$ \\
\hline$n \equiv 10(\bmod 12)$ & $\frac{2 n-2}{3}$ & $\frac{2 n+1}{3}$ & $\frac{2 n+1}{3}$ \\
\hline$n \equiv 2(\bmod 12)$ & $\frac{2 n-1}{3}$ & $\frac{2 n+2}{3}$ & $\frac{2 n-1}{3}$ \\
\hline
\end{tabular}

Table 13. Vertex label

Case 2. The triangle starts from $u_{2}$ and end with $u_{n-1}$. In this case $\left|V\left(D A T_{n}\right)\right|=2 n-2$ and $\left|E\left(D A T_{n}\right)\right|=3 n-5$. First we consider the path vertices $u_{i}$ as in the labels $1,3,2,2$ to the first four path vertices $u_{1}, u_{2}, u_{3}, u_{4}$ respectively. Then we assign the labels $1,3,2,2$ to the next four path vertices $u_{5}, u_{6}, u_{7}, u_{8}$ respectively. Proceeding like this assign the label to the next four vertices and so on. Clearly in this process the vertex $u_{n}$ received the label 2 or 3 according as $n \equiv 0(\bmod 4)$ or $n \equiv 2(\bmod 4)$. Next we move to the vertices $v_{i}$. Assign the labels $1,2,3,2,3,1$ to the six vertices $v_{1}, v_{2}, v_{3}, v_{4}, v_{5}, v_{6}$ respectively. Then we assign the labels $1,2,3,2,3,1$ to the next six vertices $v_{7}, v_{8}, v_{9}, v_{10}, v_{11}, v_{12}$ respectively. Continuing this process assign the label to the next six vertices and so on. If all the vertices of $v_{i}$ are labeled then we stop the process. Otherwise there are some non labeled vertices are exist. If the number of non labeled vertices are less than or equal to 5 then assign the labels $1,2,3,2,3$ to the non labeled vertices. If there are four non labeled vertices are exist then assign the labels $1,2,3,2$ to he non labeled vertices. If the number of non labeled vertices are three then assign the labels $1,2,3$ to the non labeled vertices. If it is two then assign the labels 1,2 to the non labeled vertices. If only one non labeled vertex is exist then assign the label 1 only. Now we consider the vertices $w_{i}$. Assign the label to the vertices $w_{6 i+2}, w_{6 i+5}, w_{6 i+3}$ for all the values of $i=0,1,2, \ldots$ Then assign the labels 3 to the vertices $w_{6 i+4}$ for $i=0,1,2,3, \ldots$ For all the values of $i=1,2,3 \ldots$ assign the label 3 to the vertices $w_{6 i}$ and $w_{6 i+1}$. The edge condition is $e_{f}(0)=\frac{3 n-4}{2}$ and $e_{f}(1)=\frac{3 n-6}{2}$. The vertex condition is given in table table 14.

Case 3. The triangle starts from $u_{2}$ and end with $u_{n}$.

It is clear in this case $\left|V\left(D A\left(T_{n}\right)\right)\right|=2 n-1$ and $\left|E\left(D A\left(T_{n}\right)\right)\right|=3 n-3$. Assign the label 1 to the path vertices $u_{12 i+1}$ and $u_{12 i+2}$ for all the values of $i=0,1,2,3, \ldots$ Next we assign the label 2 to the path vertices $u_{12 i+3}, u_{12 i+4}, u_{12 i+7}, u_{12 i+8}$ and $u_{12 i+11}$ for $i=0,1,2,3, \ldots$ For 


\begin{tabular}{|l|l|l|l|}
\hline Value of $\mathrm{n}$ & $v_{f}(1)$ & $v_{f}(2)$ & $v_{f}(3)$ \\
\hline$n \equiv 4,10(\bmod 12)$ & $\frac{2 n-2}{3}$ & $\frac{2 n-2}{3}$ & $\frac{2 n-2}{3}$ \\
\hline$n \equiv 6(\bmod 12)$ & $\frac{2 n}{3}$ & $\frac{2 n-3}{3}$ & $\frac{2 n-3}{3}$ \\
\hline$n \equiv 8(\bmod 12)$ & $\frac{2 n-1}{3}$ & $\frac{2 n-1}{3}$ & $\frac{2 n-4}{3}$ \\
\hline$n \equiv 0(\bmod 12)$ & $\frac{2 n-3}{3}$ & $\frac{2 n}{3}$ & $\frac{2 n-3}{3}$ \\
\hline$n \equiv 2(\bmod 12)$ & $\frac{2 n-1}{3}$ & $\frac{2 n-4}{3}$ & $\frac{2 n-1}{3}$ \\
\hline
\end{tabular}

Table 14. Vertex label

the values of $i=1,2,3, \ldots$ assign the label 2 to the path vertices $u_{12 i}$. Then we assign the label 3 to the path vertices $u_{12 i+5}, u_{12 i+6}, u_{12 i+9}$ and $u_{12 i+10}$ for all the values of $i=0,1,2,3, \ldots$ Now we consider the vertices $v_{i}$ and $w_{i}$. Assign the labels $3,2,1,1,2,3$ to the first six vertices $v_{1}, v_{2}, v_{3}, v_{4}, v_{5}, v_{6}$ respectively. Then we assign the labels $3,2,1,1,2,3$ to the next six vertices $v_{7}, v_{8}, v_{9}, v_{10}, v_{11}, v_{12}$ respectively. Proceeding like this assign the label to the next six vertices and so on. The last six vertices $v_{n-5}, v_{n-4}, v_{n-3}, v_{n-2}, v_{n-1}, v_{n}$ are labeled by $3,2,1,1,2,3$. Consider the vertices $w_{i}$. Assign the label to the vertices $w_{i}$ as in case 1 . The vertex condition of this case is $e_{f}(0)=e_{f}(1)=\frac{3 n-3}{2}$. The edge condition of this case is given by table 15 .

\begin{tabular}{|l|l|l|l|}
\hline Value of $\mathrm{n}$ & $v_{f}(1)$ & $v_{f}(2)$ & $v_{f}(3)$ \\
\hline$n \equiv 1(\bmod 6)$ & $\frac{2 n+1}{3}$ & $\frac{2 n-2}{3}$ & $\frac{2 n-2}{3}$ \\
\hline$n \equiv 3(\bmod 6)$ & $\frac{2 n}{3}$ & $\frac{2 n-3}{3}$ & $\frac{2 n}{3}$ \\
\hline$n \equiv 5(\bmod 6)$ & $\frac{2 n-1}{3}$ & $\frac{2 n-1}{3}$ & $\frac{2 n-1}{3}$ \\
\hline
\end{tabular}

Table 15. Vertex label

Case 4. The triangle starts from $u_{2}$ and end with $u_{n-1}$.

This case is similar to case 3 .

Finally we look into the graph double alternate quadrilateral snake. Double alternate quadrilateral snake $D A Q_{n}$ consists of two alternate quadrilateral snake that have a common path. That is it is obtained from a path $u_{1} u_{2} \ldots u_{n}$ joining $u_{i}$ and $u_{i+1}$ (alternatively) to new verticces $v_{i}, x_{i}$ and $w_{i}, y_{i}$ respectively and adding the edges $v_{i} w_{i}$ and $x_{i} y_{i}$.

Theorem 2.9. All double alternate quadrilateral snakes are 3-difference cordial graphs.

Proof. Case 1. The squares starts from $u_{1}$ and end with $u_{n}$.

In this case $\left|V\left(D A Q_{n}\right)\right|=3 n$ and $\left|E\left(D A Q_{n}\right)\right|=4 n-1$. We consider the path vertices $u_{i}$. Assign the labels $1,1,3,3$ to the first four path vertices $u_{1}, u_{2}, u_{3}, u_{4}$. Then we sign the labels $1,1,3,3$ to the next four path vertices $u_{5}, u_{6}, u_{7}, u_{8}$. Continuing this way we assign the label to the next four vertices and so on. Clearly in this process the last vertex $u_{n}$ received the label 3 or 1 according as $n \equiv 0(\bmod 4)$ or $n \equiv 2(\bmod 4)$. Now we move to the vertices $v_{i}$ and $w_{i}$. Assign the labels 2,1 to the first two vertices $v_{1}$ and $v_{2}$. Then we assign the labels 2,1 to the next two vertices and so on. Proceeding like this, we assign the labels to the next two vertices and so on. Clearly the last verte $v_{n-1}$ received the label 1 or 2 according as $n \equiv 0(\bmod 4)$ or $n \equiv 2(\bmod 4)$. Now we assign the labels 3,2 to the two vertices $w_{1}, w_{2}$ respectively. Then we assign the labels 3,2 to 
the next two vertices $w_{3}, w_{4}$ respectively. Continuing this way we assign the label to the net two vertices and so on. Note that in this process the last vertex $w_{n-1}$ receied the label 2 or 3 according as $n \equiv 0(\bmod 4)$ or $n \equiv 2(\bmod 4)$. Next we move to the vertices $x_{i}$ and $y_{i}$. Assign the labels to the vertices $x_{i}$ and $y_{i}$ is same as assign the label to the vertices $v_{i}$ and $w_{i}$. The vertex and edge condition of this case is $v_{f}(1)=v_{f}(2)=v_{f}(3)=n$ and $e_{f}(0)=2 n-1$ and $e_{f}(1)=2 n$.

Case 2. The squares starts from $u_{1}$ and end with $u_{n-1}$. In this case $\left|V\left(D A\left(Q_{n}\right)\right)\right|=3 n-4$ and $\left|E\left(D A\left(Q_{n}\right)\right)\right|=4 n-7$. Consider the path vertices $u_{i}$. Assign the label to the vertices $u_{i}$ as in case 1 . We move to the vertices $v_{i}$ and $w_{i}$. Assign the labels 2,2 to the vertices $v_{1}$ and $w_{2}$ respectively. Then assign the labels 2,1 to the vertices $v_{2}, v_{3}$ respectively. Now we assign the labels 2,1 to the next two vertices $v_{4}, v_{5}$ respectively. Continuing this process assign the label to the next two vertices and so on. Clearly the last vertex $v_{n-1}$ received the label 1 or 2 according as $n \equiv 0(\bmod 4)$ or $n \equiv 2(\bmod 4)$. Assign the labels 3,2 to the vertices $w_{2}, w_{3}$ respectively. Then assign the labels 3,2 to the next two vertices $w_{4}, w_{5}$ respectively. Proceeding like this assign the label to the next two vertices and so on. Note that the last vertex $w_{n}$ received the label 2 or 3 according as $n \equiv 0(\bmod 4)$ or $n \equiv 2(\bmod 4)$. Next we move to the vertices $x_{i}$ and $y_{i}$. Assign the labels 2,3 to the vertices $x_{1}$ and $w_{1}$ respectively. Now we assign the label 2, 1 to the vertices $x_{2}, x_{3}$. Then we assign the labels 2,1 to the next two vertices $x_{4}, x_{5}$ respectively. Continuing like this we assign the label to the next two vertices and so on. Clearly the last vertex $x_{n}$ labeled by the integers 1 or 2 according as $n \equiv 0(\bmod 4)$ or $n \equiv 2$ $(\bmod 4)$. Assign the labels 3,2 to the vertices $y_{2}, y_{3}$ respectively. Then we assign the labels 3 , 2 to the next two vertices $y_{4}, y_{5}$ respectively. Continuing this process assign the label to the next two vertices and so on. Clearly the last vertex $y_{n-1}$ received the label 2 or 3 according as $n \equiv 0$ $(\bmod 4)$ or $n \equiv 2(\bmod 4)$. The vertex and edge condition of this case is $v_{f}(1)=n-2$ and $v_{f}(2)=v_{f}(3)=n-1$ and $e_{f}(0)=2 n-3$ and $e_{f}(1)=2 n-4$.

Case 3. The squares starts from $u_{2}$ and end with $u_{n}$. In this case $\left|V\left(D A\left(Q_{n}\right)\right)\right|=3 n-2$ and $\left|E\left(D A\left(Q_{n}\right)\right)\right|=4 n-4$. Consider the path vertices $v_{i}$. Assign the label 1 to the vertex $u_{1}$. Next we assign the labels $3,3,1,1$ to the next four vertices $u_{2}, u_{3}, u_{4}, u_{5}$ respectively. Then assign the labels $3,3,1,1$ to the net four vertices $u_{6}, u_{7}, u_{8}, u_{9}$ respectively. Proceeding like this assign the label to the next four vertices and so on. Note that in this case the last verte $u_{n} u_{n}$ received the label 3 or 1 according as $n \equiv 3(\bmod 4)$ or $n \equiv 1$ $(\bmod 4)$. Next we move to the vertices $v_{i}$ and $w_{i}$. Assign the labels 1,2 to the vertices $v_{1}, v_{2}$ respectively. Then we assign labels 1,2 to th next two vertices $v_{3}, v_{4}$ respectively. Proceeding this way we assign the next two verticces and so on. Clearly the last vertex $v_{n}$ received the label 1 or 2 according as $n \equiv 3(\bmod 4)$ or $n \equiv 1(\bmod 4)$. Now we move to the vertices $w_{i}$. Assign the labels 2, 3 to the vertices $w_{1}$ and $w_{2}$ respectively. Then we assign the labels 2,3 to the next two vertices $w_{3}, w_{4}$ respectively. Continuing this way assign the label to the next two vertices and so on. Note that the last vertex $w_{n-1}$ received the label 2 or 3 according as $n \equiv 3(\bmod 4)$ or $n \equiv 1(\bmod 4)$. Consider the vertices $x_{i}$ and $y_{i}$ is same as assign the label to the vertices $v_{i}$ and $w_{i}$. The vertex and edge condition of this case is $v_{f}(1)=n$ and $v_{f}(2)=v_{f}(3)=n-1$ and $e_{f}(0)=e_{f}(1)=2 n-2$. 


\section{Acknowledgement}

The authors wish to thank the anonymous referees for their careful reading and constructive comments on earlier version of this article, which resulted in better presentation of this article.

\section{References}

[1] I. Cahit, Cordial Graphs: A weaker version of Graceful and Harmonious graphs, Ars Combin., 23 (1987) 201-207.

[2] J.A. Gallian, A Dynamic survey of graph labeling, The Electronic Journal of Combinatorics, 19 (2016) \#Ds6.

[3] F. Harary, Graph theory, Addision Wesley, New Delhi (1969).

[4] R. Hasni, S. Matarneh, A. Azaizeh, Some results on cordiality labeling of generalized Jahangir graph, Indonesian Journal of Combinatorics, 1(2017),1-8.

[5] R. Ponraj, S. Sathish Narayanan and R. Kala, Difference cordial labeling of graphs, Global Journal of Mathematical Sciences: Theory and Practical, 5(2013), 185-196.

[6] R. Ponraj and M. M. Adaickalam and R.Kala, $k$-difference cordial labeling of graphs, International Journal of Mathematical Combinatorics, 2(2016), 121-131.

[7] R. Ponraj and M. M. Adaickalam, 3-difference cordial labeling of some union of graphs, Palestine Journal of Mathematics, 6(1)(2017), 202-210.

[8] R. Ponraj and M. M. Adaickalam, 3-difference cordial labeling of cycle related graphs, Journal of Algorithms and Computation, 47(2016), 1-10.

[9] R. Ponraj and M. M. Adaickalam, 3-difference cordiality of some graphs, Palestine Journal of Mathematics, 2(2017), 141-148.

[10] R. Ponraj and M. M. Adaickalam, 3-difference cordial labeling of corona related graphs, Submitted.

[11] M. A. Seoud and S. M. Salman, On difference cordial graphs, Mathematica Aeterna, 5(1) (2015), $105-124$. 